\section{Neurological soft signs in first-episode psychosis: a systematic review*}

PAOLA DAZZAN and ROBIN M. MURRAY
Neurological soft signs (NSS) are minor ('soft') neurological abnormalities in sensory and motor performance identified by clinical examination. They have been described in excess in patients with schizophrenia (Buchanan \& Heinrichs, 1989). There is still a lack of consensus on the neurodysfunctional area underlying NSS; some authors suggest that NSS reflect a failure in the integration within or between sensory and motor systems (Griffiths et al, 1998), whereas others advocate deficits in neuronal circuits involving subcortical structures (e.g. basal ganglia, brain-stem and limbic system; Heinrichs \& Buchanan, 1988). Although the presence of NSS has been documented extensively in schizophrenia as a whole, the same cannot be said for patients undergoing their first psychotic episode. This review will briefly describe the NSS that have been commonly reported in patients with schizophrenia, and then concentrate on studies that have specifically investigated NSS at the time of the first psychotic episode.

\section{NEUROLOGICAL SIGNS REPORTED IN SCHIZOPHRENIA}

The majority of investigations into patients with schizophrenia at various stages of chronicity have described an excess of neurological 'soft' abnormalities. The term 'soft' was originally used, as opposed to 'hard', to reflect the absence of any obvious localised pathological lesion underlying these signs (Tucker et al, 1974; Quitkin et al, 1976). This term has been employed, more recently, to indicate signs that do not reflect primary tract or nuclear pathology (Woods et al, 1986). Although the categorisation of neurological signs as 'soft' (e.g. frontal release and cerebellar signs) and the batteries used to measure them have varied, neurological abnormalities in schizophrenia seem to be localised to three main neurological domains: integrative sensory function; motor coordination; and motor sequencing (Table 1) (Buchanan \& Heinrichs, 1989). Deficits in integrative sensory function (possibly resulting from a parietal dysfunction) are reflected in higher rates of bilateral extinction, impaired audio-visual integration, agraphaesthesia and astereognosis (Buchanan \& Heinrichs, 1989; Griffiths et al, 1998). Deficits in motor coordination have been reported through tests of general coordination, intention tremor, finger-thumb opposition, balance and gait. Finally, poor performance in complex motor tasks (possibly resulting from a dysfunction of the frontal-basal ganglial circuitry) has been reported in tests that involve repetitive alternating hand positions, such as the fist-edge-palm, the fist-ring and the Ozeretski tests.

Abnormalities have also been reported in eye movements (pursuit and saccadic movements; Stevens, 1982) and developmental reflexes (Boks et al, 2000), particularly in signs of frontal release. Other abnormalities, such as those of primary sensory function, have been less often described.

\section{NEUROLOGICAL SOFT SIGNS IN FIRST-EPISODE PSYCHOSIS}

Investigating patients at the early stages of the illness has the following potential findings and clarifying the role of NSS as markers of cognitive dysfunction.

\section{Declaration of interest None.}

Funding from the Stanley Foundation and the Medical Research Council (UK).

* Presented in part at the European First Episode Schizophrenia Network Meeting,Whistler BC, Canada, 27 April 200I.
Table I Functional areas frequently reported as abnormal in schizophrenia, and some of the tests that can elicit disturbances in these areas

\begin{tabular}{llll}
\hline Sensory integration & Motor coordination & Motor sequencing & Primitive reflexes \\
\hline Audio-visual integration & Tandem walk & Fist-ring test & Gaze \\
Stereognosis & Rapid alternating movements & Fist-edge-palm test & Palmomental \\
Graphaestesia & Finger-thumb opposition & Oszeretski test & Snout \\
Extinction & Finger-nose test & & Grasp \\
Right-left confusion & Rhythm tapping & & \\
\hline
\end{tabular}


advantages: it can clarify whether NSS are part of a neurodysfunction that underlies schizophrenia rather than the consequence of degenerative processes; and it can elucidate whether or not they are simply a neuroleptic-induced epiphenomenon (i.e. a consequence of long-term pharmacological treatment).

In considering patients with first-onset psychosis, it is important to note that some studies have included not just patients with schizophrenia but also those with other forms of psychosis. This over-inclusiveness is unavoidable as assessments are frequently performed at the very early stages of a psychotic presentation, when a diagnosis of definite schizophrenia may still be doubtful. Not including cases until they definitely qualify for a diagnosis of schizophrenia at a later stage would mean losing potential cases of schizophrenia from the initial sample. Therefore, for the purpose of this review, we will also include studies that have evaluated first-episode psychosis as a whole.

We decided to evaluate those studies that investigated neurological function in patients with first-episode psychosis using a clinical examination that specifically reported on the neurological function of this group of patients. We performed a Medline literature search of the period between 1966 and 2001 using the following terms: PSYCHIATRY and NEURO*; SCHIZOPHRENIA and NEURO*; FIRST EPISODE and NEURO*; FIRST EPISODE and SOFT-SIGNS; SOFT-SIGNS and NSS. We also examined cross-references from the articles identified.

We identified nine studies, which are listed in Table 2.

First-episode studies of NSS can be divided into: (a) studies that have evaluated the prevalence of NSS among patients with first-episode psychosis only (i.e. without including a comparison group); (b) studies that have evaluated NSS in first-episode patients and compared them with healthy controls; (c) studies that have compared first-episode patients with subjects at high risk of developing psychosis; and finally, (d) studies that have evaluated NSS at first-episode and then at follow-up. Unfortunately, a variety of instruments have been used to evaluate neurological function, and not all studies have used a published, validated instrument, often reporting on findings derived from a 'clinical neurological assessment', which makes comparison of the results difficult (Table 2).

\section{The prevalence of NSS among first-episode psychosis patients}

Studies that evaluated patients with firstepisode psychosis have reported a high prevalence of NSS, the percentage of patients with NSS varying from 20\% (The Scottish Schizophrenia Research Group, 1987) to 97.1\% (Browne et al, 2000). The Scottish Schizophrenia Research Group (1987) used the Northwick Park Brief Neurological Assessment (Cunningham Owens \& Johnstone, 1980); abnormalities were observed particularly in plantar responses and coordination (as well as weakness in lower limbs), diminished tone in lower limbs and increased ankle and knee jerks.

The study by Browne et al (2000) is noteworthy in that information was collected using two validated scales: the Neurological Evaluation Scale (NES; Buchanan \& Heinrichs, 1989) and the Condensed Neurological Examination

Table 2 Studies included in this review, listed in alphabetical order

\begin{tabular}{|c|c|c|c|c|c|}
\hline Reference & $\begin{array}{c}\text { First-episode } \\
\text { patients } \\
n\end{array}$ & Diagnosis & $\begin{array}{l}\text { Healthy } \\
\text { controls } \\
n\end{array}$ & $\begin{array}{c}\text { High-risk } \\
\text { subjects } \\
n\end{array}$ & Evaluation scale \\
\hline Browne et al, 2000 & 56 & $\begin{array}{l}\text { Schizophrenia/schizophreniform } \\
\text { disorder }\end{array}$ & - & - & $\begin{array}{l}\text { Neurological Evaluation Scale (Buchanan } \\
\text { \& Heinrichs, 1989) } \\
\text { Condensed Neurological Examination } \\
\text { (Rossi et al, 1990) }\end{array}$ \\
\hline Carr et al, 2000 & 56 & Psychosis & - & 60 & $\begin{array}{l}\text { Neurological Evaluation Scale (Buchanan } \\
\text { \& Heinrichs, 1989) }\end{array}$ \\
\hline Flyckt et al, 1999 & 21 & Schizophrenia & 55 & - & Standard neurological examination \\
\hline Johnstone et al, 1990 & 137 & Schizophrenia & - & - & $\begin{array}{l}\text { Neurological examination (adapted from } \\
\text { Quitkin et al, 1976) }\end{array}$ \\
\hline Lawrie et al, 200 I & 30 & Schizophrenia & 35 & 152 & $\begin{array}{l}\text { Neurological Evaluation Scale (Buchanan } \\
\text { \& Heinrichs, 1989) }\end{array}$ \\
\hline Madsen et al, 1999 & $34^{\prime}$ & Schizophrenia & 20 & - & Standard neurological examination \\
\hline Rubin et al, 1994 & 45 & $\begin{array}{l}\text { Schizophrenia/schizophreniform } \\
\text { disorder }\end{array}$ & 24 & - & Standard neurological examination \\
\hline Sanders et al, 1994 & 17 & $\begin{array}{l}\text { Schizophrenia/schizophreniform } \\
\text { disorder }\end{array}$ & 15 & - & $\begin{array}{l}\text { Neurological Evaluation Scale (Buchanan } \\
\text { \& Heinrichs, 1989) }\end{array}$ \\
\hline $\begin{array}{l}\text { The Scottish Schizophrenia } \\
\text { Research Group, } 1987\end{array}$ & 49 & Schizophrenia & - & - & $\begin{array}{l}\text { Northwick Park Brief Neurological } \\
\text { Assessment (Cunningham Owens \& } \\
\text { Johnstone, 1980) }\end{array}$ \\
\hline
\end{tabular}

I. This study evaluated 34 patients with schizophrenia and 29 patients with other psychiatric disorders at the time of their first admission, and 20 healthy controls. After 5 years, the authors re-investigated 18 patients with schizophrenia, II patients with other psychiatric disorders and 10 healthy controls. 
(CNE; Rossi et al, 1990). The results of the two scales were correlated strongly; at least one NSS (defined as one NES item rated 2) was displayed by $97.1 \%$ of patients, with $63 \%$ showing at least two NSS (defined as 2 or more NES items rated 2).

\section{The prevalence of NSS in patients with first-episode psychosis in comparison with healthy controls}

The prevalence of NSS in healthy subjects has been reported to vary from 5\% (Hertzig \& Birch, 1968; Rochford et al, 1970) to more than 50\% (Kennard, 1960; Cox \& Ludwig, 1979), the proportion reported being mainly a function of the measure used. It is therefore advantageous to compare the rates of NSS in patients with first-episode psychosis with those of healthy controls, using identical assessment procedures.

Overall, there is a higher prevalence of NSS in first-episode patients compared with healthy controls. In particular, significantly higher total rates of NSS in patients with first-episode schizophrenia or schizophreniform disorder have been reported (Rubin et al, 1994; Gupta et al, 1995). Moreover, first-episode patients also show a worse performance in: each of the NES sub-scales (Sanders et al, 1994); facial expression (Flyckt et al, 1999); hypokinesia (Flyckt et al, 1999); hand tremor (Flyckt et al, 1999); neck tonus (Flyckt et al, 1999); and cerebellar signs (Rubin et al, 1994).

In the study by Flyckt et al (1999), patients with first-episode schizophrenia and patients with chronic schizophrenia were compared as a whole with healthy controls. The patient group reported significantly higher NSS scores than the controls, and although the absolute figures were not reported separately for chronic and firstepisode patients, the authors commented that there were no differences between first-episode and chronic patients.

We can conclude that the rates of neurological signs in patients at their firstepisode of psychosis are already higher than those of healthy controls. Furthermore, these rates are comparable with those described in studies of schizophrenia as a whole, which have reported an excess of NSS, particularly for motor coordination, in $59-92 \%$ of patients (Tucker et al, 1975). A higher consistency in rates could be achieved if the assessors were blind to the subject's status, a task easier to achieve if patients with other psychiatric disorders represent the control group. Considering that NSS rates similar to those observed in schizophrenia have been reported in patients with personality disorder (Rochford et al, 1970), it seems important that subjects with schizophrenia are also compared with subjects affected by other putative neurodevelopmental disorders (e.g. obsessive-compulsive disorder, attention-deficit hyperactivity disorder).

\section{The prevalence of NSS in patients with first-episode psychosis in comparison with high-risk subjects}

Having established that an excess of NSS is already present in the early phases of psychosis, the next question is whether or not this excess pre-dates the onset of psychosis and thus could be a vulnerability marker for psychosis. Impairments of motor development and fine motor coordination have been observed in children from cohort studies who later go on to develop schizophrenia (Crow et al, 1995; Cannon et al, 1999). The presence of these abnormalities suggests that neurological dysfunction could reflect a neurodevelopmental abnormality that puts the individuals at risk of later schizophrenia.

Much attention has been devoted to asymptomatic subjects at high genetic risk of schizophrenia, with similar abnormalities having been reported (Rieder \& Nichols, 1979; Fish et al, 1992; Marcus et al, 1993). In one study (Carr et al, 2000), subjects at first-episode psychosis were compared with high-risk asymptomatic individuals, and were reported as showing similar NSS rates. In another study, although first-episode patients showed more NSS than high-risk subjects, highrisk subjects had an excess of NSS compared with healthy controls, specifically for signs of sensory integration (Lawrie et al, 2001).

These findings suggest that neurological dysfunction observed in first-episode patients is at least in part related to the pathogenesis underlying the illness, and can be observed in association with an increased risk of the disorder, even before the onset of a full-blown psychotic illness (Lawrie et al, 2001). This is consonant with reports of neurological abnormalities not only in the relatives of patients with schizophrenia (Kinney et al, 1986; Griffiths et al,
1998; Niethammer et al, 2000), but also in the offspring of parents with schizophrenia (Fish \& Hagin, 1973; Marcus et al, 1985). In these latter cases, the neurological abnormalities were similar to those described in adult patients: problems in coordination, motor dysfunction and sensory integration (Heinrichs \& Buchanan, 1988).

\section{The temporal stability of NSS following the first psychotic episode}

If we accept the above evidence that NSS are already present at first onset and indeed in vulnerable asymptomatic high-risk individuals, the question of their temporal stability can only be addressed by followup studies.

The only study that we have identified that investigated NSS at both first onset and at follow-up is that of Madsen et al (1999). At the time of their first psychotic episode, patients showed a significantly higher total rate of neurological abnormalities than healthy controls. Five years later, the difference between patients with schizophrenia and healthy controls was increased, especially for frontal, corticospinal and temporo-parietal functions. By contrast, patients with other psychiatric disorders showed a reduction in the number of neurological abnormalities at follow-up, indicating that their neurological function improved with remission, although they still showed significantly higher rates of neurological abnormalities than healthy controls, mainly in frontal lobe functions. Thus, this study suggests a progression of neurological dysfunction comparable to that previously reported in chronic patients with schizophrenia (Torrey, 1980), although treatment effects could have contributed to this.

Thus NSS, particularly in motor coordination and in developmental reflexes, are already present in patients at very early stages of the illness. Furthermore, the neurological anomalies shown by patients undergoing their first episode of schizophrenia do not improve with time and may actually deteriorate. A similar neurological dysfunction is present even in highrisk subjects without psychosis. This is in accord with previous evidence that an impairment of motor development and fine motor coordination can predict schizophrenia-spectrum disorders in adulthood (Crow et al, 1995), and may be part of a genetically transmitted vulnerability to develop schizophrenia, as suggested by 
studies on children at high genetic risk (Rieder \& Nichols, 1979; Fish et al, 1992; Marcus et al, 1993).

\section{NEUROLOGICAL SOFT SIGNS AND FAMILIALITY}

Therefore, investigators have studied the relationship between NSS and genetic vulnerability to this illness. An excess of NSS (especially of integrative signs) has been reported in patients with a positive family history for schizophrenia, in comparison with those without such a history (Walker \& Shaye, 1982; Griffiths et al, 1998), and indeed healthy relatives of patients with schizophrenia (Griffiths et al, 1998; Ismail et al, 1998; Niethammer et al, 2000).

The majority of the first-episode studies reviewed did not evaluate the relationship between neurological function and familiality. Three studies (Flyckt et al, 1999; Madsen et al, 1999; Lawrie et al, 2001) evaluated family history or genetic loading and neurological function and reported no association between prevalence of NSS and family history. However, a relationship was reported between family history and a specific laterality pattern in psychomotor performance in one study (Flyckt et al, 1999), and family history and progression of neurological dysfunction in another (Madsen et al, 1999).

In the study by Madsen et al (1999), information was obtained on psychiatric disorders in the first- and second-degree relatives. Although there was no association between a positive family history of psychosis and NSS at first presentation, the patients with a history of psychotic disorder in first-degree relatives showed a significant neurological deterioration at follow-up.

The report of no association between positive family history and neurological dysfunction in these first-episode studies is in contrast with previous observation of a relationship between the two reported in patients at various illness stages. However, it is possible that, if a positive family history is associated with more progression in neurological dysfunction, studies of patients in more advanced stages of schizophrenia could have had more chances of identifying this association, less evident in the initial stages of the illness. It is, however, difficult to draw a conclusion from the few studies reported.

\section{LATER ALITY AND CEREBRAL DOMINANCE}

To date, research has not identified an unequivocal laterality pattern of neurological signs in schizophrenia in general (and by implication a lesion of a particular hemisphere). Some studies have reported a predominance of neurological abnormalities on the right side of the body (Torrey, 1980; Caligiuri \& Lohr, 1994), but there are conflicting reports of higher scores for NSS on the left side of the body (Niethammer et al, 2000).

The first-onset study of Browne et al (2000) evaluated the relationship of mixedhandedness (measured with the Edinburgh Inventory; Oldfield, 1971) to NSS. In the Edinburgh Inventory, subjects are asked to perform 10 common activities and a laterality quotient is then calculated. The authors reported that narrowly defined mixed-handed subjects (two or more discrepancies in hand preference) performed worse on both the NES and the CNE examinations, in comparison with lateralised individuals. Although the number of mixed-handed subjects was small, this raises the possibility that individuals whose handedness is not lateralised have poorer motor coordination than those with a lateralised hand preference. This finding should be interpreted along with the reported excess of mixed-handedness in patients with psychosis, and especially with schizophrenia (Cannon et al, 1995; Malesu et al, 1996; Orr et al, 1999).

Mixed-handedness in schizophrenia has been postulated by Crow (1997) to reflect an abnormality of cerebral dominance. Possible relevant information comes from our preliminary report (Dazzan et al, 2001) of a worse neurological performance (especially for coordination and sequencing) on the left side of the body in a group of 145 subjects with first-episode psychosis. A common neurodevelopmental fault can underlie both the excess of mixed-handedness in schizophrenia and that of an abnormal neurological function (possibly lateralised to one side of the body), which was particularly marked in these subjects.

The study by Flyckt et al (1999) evaluated both patients with schizophrenia (some at first-episode and some in more advanced stages) and their parents. There were no differences in NSS between patients positive or negative for family history. However, probands and relatives were also asked to perform the fingertapping test, a test of psychomotor performance. A significant difference was observed in the laterality pattern of patients and parents who were family-history positive, compared with patients and parents who were family-history negative. More specifically, in this test, patients and parents who were family-history positive improved their performance with the preferred hand, whereas the family-history negative parents neither significantly improved nor decreased their performance with the preferred hand over time. Considering that the finger-tapping test can provide information on cerebral laterality (Gorynia \& Uebelhack, 1992), and that an abnormal pattern of hemispheric asymmetry has been reported in families multiply affected by schizophrenia (Sharma et al, 1999), it is possible that the association between family history and a specific laterality pattern could reflect an inherited predisposition to this illness.

\section{ROLE OF ANTIPSYCHOTIC MEDICATION IN NEUROLOGICAL PERFORMANCE}

Could side-effects of antipsychotic medication in general, and tardive dyskinesia in particular, explain the excess of NSS in subjects with psychosis? No association between past and current neuroleptic exposure and presence of NSS has been found (Ismail et al, 1998). Indeed, most studies have failed to find any association between tardive dyskinesia and NSS (Wegner et al, 1985; King et al, 1991; Mohr et al, 1996). In an attempt to control for the possible contribution of neuroleptic medication to NSS, scales for extrapyramidal symptoms, akathisia and tardive dyskinesia have been used in studies of NSS; results have shown that these neurological abnormalities cannot be simply considered a medication effect (Griffiths et al, 1998).

However, more definitive information on the effect of antipsychotics on neurological function can be obtained by studying neuroleptic-naïve subjects who have never been exposed to pharmacological treatment, or subjects at their first psychotic episode, who have not been exposed to long-term pharmacological treatment.

Studies on neuroleptic-naïve firstepisode patients consistently report higher 
NSS rates in these subjects than in healthy controls. Madsen et al (1999) compared neuroleptic-naïve subjects with firstepisode schizophrenia or schizophreniform disorder with healthy control subjects; neuroleptic-naïve patients showed significantly higher rates of neurological abnormalities than controls. Flyckt et al (1999) examined first-episode and chronic patients together. There were no differences in NSS between medicated and non-medicated subjects, except for facial expression. There was no correlation between NSS and either the daily dosage of medication or the length of treatment.

Browne et al (2000) reported that the rates of NSS did not differ significantly in neuroleptic-naïve or neuroleptic-treated patients, with $97.1 \%$ of neuroleptic-naïve subjects with psychosis showing at least one neurological sign. This rate is much higher than the rate reported from other studies (Gupta et al, 1995), and could have been related to differences in the sensitivity of the assessment and the scoring guidelines used. For example, in the study by Gupta $e t$ al (1995) the assessment did not include signs of frontal lobe dysfunction, which are considered the tests most likely to identify the motor disturbance present in schizophrenia (Manschreck et al, 1981). This is also supported by the findings of Cuesta et al (1996), who evaluated frontal signs through motor coordination and sequencing of complex motor tasks and reported rates of NSS in neuroleptic-naïve patients as high as those of Browne and colleagues.

Indirect evidence that NSS are not related to neuroleptic treatment also comes from the study of Lawrie et al (2001). They reported how the excess of sensory integration signs was similar in both firstepisode patients and high-risk subjects, and significantly higher than in healthy controls, suggesting that NSS represent a neurodevelopmental risk factor for schizophrenia rather than being the consequence of having been exposed to neuroleptic treatment.

Surprisingly, some authors have proposed a hypothetical protective effect of antipsychotics on neurological dysfunction. For example, in the follow-up study by Madsen et al (1999), there was an increase in NSS in patients with schizophrenia 5 years after their first present ation; this increase was more marked in those patients who had been free of medication for the time of the entire follow-up. Signs related to the corticospinal tract were over-represented. The authors reported how the neuroleptic load in non-responder patients was inversely related to change in total neurological abnormalities. It is also possible that the positive effect observed is related to the efficacy of antipsychotics on the clinical presentation.

Thus, first-episode studies demonstrate that the neurological dysfunction observed could not be interpreted as the consequence of neuroleptic medication, and one study suggests that antipsychotics may, directly or indirectly, improve the baseline neurological dysfunction. However, it is important that future studies addressing this issue include in their battery signs of frontal lobe dysfunction, because these best reflect the motor abnormalities typical of schizophrenia, rather than those related to neuroleptic use.

\section{NEUROLOGICAL SOFT SIGNS AND DEMOGRAPHIC CHARACTERISTICS}

Studies of patients at various stages of schizophrenia other than at first-episode have generally failed to report any consistent association between neurological abnormalities and socio-demographic characteristics of patients (Ismail et al, 1998). However, there have been isolated reports of NSS being increased especially in male patients with schizophrenia (Heinrichs \& Buchanan, 1988), or more dependent on age and duration of illness (Lane et al, 1996; Blyler et al, 1997; Malla et al, 1997). When trying to disentangle whether these associations are part of the underlying aetiopathological process, or simply its epiphenomena, it is important to control for these variables.

The studies of patients with firstepisode psychosis reviewed here reported no relationship between the presence of NSS and age (Sanders et al, 1994), but possible relationships have been reported with male gender (The Scottish Schizophrenia Research Group, 1987; Madsen et al, 1999), lower education (Rubin et al, 1994; Browne et al, 2000), and lower socio-occupational outcome (Johnstone et al, 1990).

Male patients were described as having a significant increase in the number of neurological abnormalities 5 years after onset in a follow-up study by Madsen et al (1999). In this sample, males were also more likely to have been subjected to maternal obstetric complications and a non-remitting course of the illness. In the Scottish Schizophrenia Research Group's study (1987), most of the non-responders were males with neurological impairment. It is possible that the excess of neurological abnormalities in male patients, together with an increased risk of neurodevelopmental damage and of a generally more severe illness (Castle \& Murray, 1991; Bullmore et al, 1995), is part of a greater vulnerability of the developing male brain to environmental insults (Murray, 1994).

The presence of a neurodevelopmental abnormality may also explain the reported association between lower educational achievement and higher rates of NSS in first-episode studies (Rubin et al, 1994). However, the evidence derived from the study by Browne et al (2000) is indirect. In fact, in their study a significant difference in years of education was present between mixed-handed and lateralised patients, with the mixed-handed patients having a significantly lower number of years of completed education. As mixedhanded patients showed more neurological abnormalities, the authors speculated that neurological abnormalities were also associated with lower years of education. These results should be interpreted cautiously; it is likely that patients with more severe forms of the disease, and with higher rates of neurological abnormalities, would have found it more difficult to continue their education.

Nevertheless, the above results are consonant with evidence of an association between IQ and neurological abnormalities, specifically between IQ and signs of sensory integration (Kennard, 1960; Mosher et al, 1971; Arango et al, 1999). It has been suggested that the concomitant presence of NSS and cognitive deficits in schizophrenia could reflect a diffuse, generalised brain disorder (Kolakowska et al, 1985; King et al, 1991; Flashman et al, 1996). The line between some NSS and selected neuropsychological tests is often difficult to draw: evaluating these together could provide comprehensive information on a range of regional neurological dysfunctions. Even just IQ, which has been extensively evaluated in previous studies, has not been frequently examined in first-episode studies on NSS. 


\section{NEUROLOGICAL SOFT SIGNS AND BRAIN STRUCTURAL ABNORMALITIES}

Clarifying the relationship between brain structure and neurological dysfunction could point to the anatomical substrates of NSS present in schizophrenia. Unfortunately, only a few studies of chronic patients have examined this relationship. The presence of NSS has been associated with an enlargement of cerebral ventricles (Weinberger \& Wyatt, 1982), and with smaller brain areas (DeMyer et al, 1988), whereas no correlation has been found between NSS and the calculated ratio between the width of the ventricles and the brain (Kolakowska et al, 1985).

Among first-episode studies, the only one that evaluated this relationship was the computerised tomography (CT) study by Rubin et al (1994). This study reported an association between NSS and shorter brain length and wider left Sylvian fissure, together with a tendency for patients with more neurological abnormalities to have smaller brain volume, more cerebrospinal fluid in the sulci and cisterna on the brain surface, increased width of the right Sylvian fissure and smaller temporal horn volume. As in previous studies (Kolakowska et al, 1985), there was no indication that NSS were associated with greater volume of lateral ventricles. The findings of this study suggest that neurological abnormalities could be associated with cortical rather than subcortical lesions. More studies with large samples, investigating the anatomical correlates of neurological signs specifically selected to explore regional function/ dysfunction, are needed before firm conclusions can be drawn.

\section{RELATIONSHIP BETWEEN NSS AND THE PSYCHOPATHOLOGYAND COURSE OF SCHIZOPHRENIA}

Previous studies on patients at different stages of their illness have asked whether neurological abnormalities are associated with a specific symptom profile or course of the schizophrenic illness. Some studies have reported a relationship between NSS and different subtypes of schizophrenia, such as chronic $v$. acute schizophrenia (Torrey, 1980) and disorganised $v$. nondisorganised schizophrenia (Schroder et al, 1991). Neurological abnormalities have also been associated with total number of psychiatric symptoms (Tucker \& Silberfarb, 1978), thought disorder (Tucker et al, 1974, 1975; Manschreck et al, 1981, 1982), negative symptoms (Caligiuri \& Lohr, 1994; Wong et al, 1997) and emotional stability (Quitkin et al, 1976). By contrast, other studies have found no association between NSS and positive symptoms (Kolakowska et al, 1985), or paranoid/non-paranoid schizophrenia (Manschreck \& Ames, 1984).

Similar conflicting results have also characterised studies that evaluated the relationship between psychopathology and NSS in first-episode psychosis. For example, Browne et al (2000) described an association between NSS and total symptom severity and positive symptoms whereas others have reported no association with global measures of psychopathology (Sanders et al, 1994), or with positive and negative dimensions of schizophrenia (Flyckt et al, 1999). A possible reason for the inconsistency of these results lies in the different scale used to measure NSS. For example, Flyckt et al (1999) did not use a scale that included factors involving attention and initiative. As Browne et al (2000) suggested, the correlation between total NSS and positive symptoms may reflect attentional deficits secondary to untreated symptoms. Not only is the number of first-episode studies small, but some studies also lacked sufficient power to evaluate such associations.

The potential association between the presence of neurological signs and a more chronic and severe form of the illness has also been investigated by studies on patients at different stages of the illness. This has been supported by the association of NSS with young age at onset (Torrey, 1980), a more chronic course (Torrey, 1980), longer index hospitalisation (Rochford et al, 1970) and impaired premorbid functioning (Quitkin et al, 1976; Kolakowska et al, 1985). However, some of these findings have not been corroborated by other studies, in which no association was found with age at onset (Kolakowska et al, 1985), poor premorbid functioning (Torrey, 1980; Kolakowska et al, 1985), number of total hospitalisations in a 3 -year follow-up (Tucker $\&$ Silberfarb, 1978) and lifetime hospitalisations (Mosher et al, 1971).

The majority of first-episode studies report no correlation between NSS and age at onset (Madsen et al, 1999), duration of untreated psychosis (Madson et al, 1999; Browne et al, 2000), global assessment of functioning (Sanders et al, 1994), and occupational outcome (Johnstone et al, 1990). It is possible that factors such as occupational outcome and global assessment of functioning are worse in more advanced phases of the illness, and are therefore not associated with neurological dysfunction in the initial stages. However, two studies described an association between NSS and both poorer premorbid social adjustment (Browne et al, 2000) and number of in-patient days (Johnstone $e t$ al, 1990). These associations could be related to the fact that higher rates of signs are part of a more severe clinical picture, which could for example explain the longer time spent in hospital; it is also possible that this is reflected in longer pharmacological treatment, which could in turn give rise to more NSS.

In conclusion, the studies reviewed confirm that an excess of NSS is already evident in patients suffering their firstepisode of schizophrenia or psychosis, and indeed in high-risk subjects without psychosis. Neurological performance appears to be worse in the areas of motor coordination and sequencing, in sensory integration and in developmental reflexes. These minor neurological anomalies are found particularly in males and in subjects with lower education, and possibly in those with a more severe clinical picture. The presence of neurological abnormalities cannot be explained away as a consequence of neuroleptic use, although higher NSS scores are observed in those first-episode subjects on neuroleptic treatment. For an optimal differentiation of the motor disturbance typical of schizophrenia from the sideeffects of neuroleptic treatment, it is important that signs of frontal lobe dysfunction are included in the assessment.

First-episode studies also support an association between neurological signs and a specific pattern of laterality, as suggested by their particular excess in mixed-handed subjects, and by some preliminary reports of their lateralisation to one side of the body. This abnormal laterality pattern in psychomotor performance is more frequently accompanied by a positive family history of psychosis, and is present in relatives without psychosis that are positive for family history. It is possible that this could be related to the evidence that, as part of the genetic vulnerability to develop schizophrenia, the cerebral hemispheres develop less asymmetrically. These findings 
support the notion that neurological signs mainly result from a genetic vulnerability rather than neurodegeneration, or the consequences of the illness (pharmacological treatment, institutionalisation). Future studies should therefore attempt to identify whether any NSS is specific and discriminative of schizophrenia, as opposed to other psychiatric disorders.

\section{ACKNOWLEDGEMENTS}

We thank Drs Ken G. Orr and Brian T. Toone for their useful comments on the manuscript. We are also grateful to the Stanley Foundation and Medical Research Council (UK) for financial support.

\section{REFERENCES}

Arango, C., Bartko, J. J., Gold, J. M., et al (1999) Prediction of neuropsychological performance by neurological signs in schizophrenia. American journal of Psychiatry, 156, 1349-1357.

Blyler, C. R., Maher, B. A., Manschreck, T. C., et al (1997) Line drawing as a possible measure of lateralized motor performance in schizophrenia. Schizophrenia Research, 26, 15-23.

Boks, M. P., Russo, S., Knegtering, R., et al (2000) The specificity of neurological signs in schizophrenia: a review. Schizophrenia Research, 43, 109-116.

\section{Browne, S., Clarke, M., Gervin, M., et al (2000)} Determinants of neurological dysfunction in first episode schizophrenia. Psychological Medicine, 30, |433-144|.

\section{Buchanan, R.W. \& Heinrichs, D. W. (1989)}

The neurological evaluation scale (NES): A structured instrument for the assessment of neurological signs in schizophrenia. Psychiatry Research, 27, 335-350.

Bullmore, E., Brammer, M., Harvey, l., et al (1995) Cerebral hemispheric asymmetry revisited: Effects of handedness, gender and schizophrenia measured by radius of gyration in magnetic resonance images. Psychological Medicine, 25, 349-363.

Caligiuri, M. P. \& Lohr, J. B. (1994) A disturbance in the control of muscle force in neuroleptic-naive schizophrenic patients. Biological Psychiatry, 35, 104-III.

Cannon, M., Byrne, M., Cassidy, B., et al (1995) Prevalence and correlates of mixed-handedness in schizophrenia. Psychiatry Research, 59, 119-125.

_ , Jones, P., Huttunen, M. O., et al (1999) Motor co-ordination deficits as predictors of schizophrenia among Finnish school children. Human

Psychopharmacology: Clinical and Experimental, 14 491-497

Carr, V., Halpin, S., Lau, N., et al (2000) A risk facto screening and assessment protocol for schizophrenia and related psychosis. Australian and New Zealand Journal of Psychiatry, 34 (suppl.), SI70-SI80.

Castle, D. J. \& Murray, R. M. (1991) The

neurodevelopmental basis of sex differences in

schizophrenia. Psychological Medicine, 21, 565-575.

Cox, S. M. \& Ludwig, A. M. (1979) Neurological soft signs and psychopathology. I. Findings in schizophrenia. Journal of Nervous and Mental Disease, 167, $161-165$

\section{CLINICAL IMPLICATIONS}

- A neurological dysfunction is already evident in patients having their first-episode of schizophrenia or psychosis, and is not the result of illness progression.

- The presence of neurological abnormalities in schizophrenia cannot be explained away as a consequence of neuroleptic use.

- The presence of a neurological dysfunction in schizophrenia may contribute to identifying subjects at risk of a poor prognosis, who may benefit from early intervention.

\section{LIMITATIONS}

The number of studies investigating neurological performance in first-episode psychosis is still small.

- A variety of instruments have been used to evaluate neurological function, and this makes comparison of the results difficult.

- The majority of first-episode psychosis studies did not investigate neurological performance separately for the two sides of the body, hampering the understanding of the role of lateralisation in neurological dysfunction.

PAOLA DAZZAN, MRCPsych, ROBIN M. MURRAY, FRCPsych, Institute of Psychiatry, London, UK

Correspondence: Dr Paola Dazzan, Division of Psychological Medicine, Institute of Psychiatry, De Crespigny Park, London SE5 8AF. Tel: +44 (0) 207848 0590; fax: +44 (0) 207 70l 9044; e-mail: spcbpad@iop.kcl.ac.uk

Crow, T. J. (1997) Is schizophrenia the price that Homo sapiens pays for language? Schizophrenia Research, $\mathbf{2 8}$ $|27-| 4 \mid$.

_ , Done, D. J. \& Sacker, A. (1995) Childhood precursors of psychosis as clues to its evolutionary origins. European Archives of Psychiatry and Clinical Neuroscience, 245, 61-69.

\section{Cuesta, M. J., Peralta, V. \& de Leon, J. (1996)} Neurological frontal signs and neuropsychological deficits in schizophrenic patients. Schizophrenia Research 20, $15-20$

Cunningham Owens, D. G. \& Johnstone, E. C. (1980) The disabilities of chronic schizophrenia - their nature and the factors contributing to their development. British Journal of Psychiatry, 136, 384-395.

Dazzan, P., Morgan, K. D., Orr, K. G., et al (200I) Lateralisation of neurological soft signs in the AESOP (London) first-onset psychosis study. Schizophrenia Research, 49 (suppl.), 104.

DeMyer, M. K., Gilmor, R. L., Hendrie, H. C., et al (1988) Magnetic resonance brain images in schizophrenic and normal subjects: influence of diagnosis and education. Schizophrenia Bulletin, I4, 21-37.

Fish, B. \& Hagin, R. (1973) Visual-motor disorders in infants at risk for schizophrenia. Archives of General Psychiatry, 28, 900-904.

_, Marcus, J., Hans, S. L., et al (1992) Infants at risk for schizophrenia: Sequelae of a genetic neurointegrative defect: A review and replication analysis of pandysmaturation in the Jerusalem infant development study. Archives of General Psychiatry, 49, 221-235.
Flashman, L. A., Flaum, M., Gupta, S., et al (1996) Soft signs and neuropsychological performance in schizophrenia. American Journal of Psychiatry, I53, 526-532.

Flyckt, L., Sydow, O., Bjerkenstedt, L., et al (1999) Neurological signs and psychomotor performance in patients with schizophrenia, their relatives and healthy controls. Psychiatry Research, 86, II3-129.

Gorynia, I. \& Uebelhack, R. (1992) Functional motor asymmetries correlated with clinical findings in unmedicated schizophrenic patients. European Archives of Psychiatry and Clinical Neuroscience, 242, 39-45.

Griffiths, T. D., Sigmundsson, T., Takei, N., et al (1998) Neurological abnormalities in familial and sporadic schizophrenia. Brain, |2I, |9|-203.

Gupta, S., Andreasen, N. C., Arndt, S., et al (1995) Neurological soft signs in neuroleptic-naive and neuroleptic-treated schizophrenic patients and in normal comparison subjects. American journal of Psychiatry, I52, 191-196.

Heinrichs, D.W. \& Buchanan, R.W. (1988) Significance and meaning of neurological signs in schizophrenia. American Journal of Psychiatry, 145, II-18.

Hertzig, M. E. \& Birch, H. G. (1968) Neurologic organization in psychiatrically disturbed adolescents. A comparative consideration of sex differences. Archives of General Psychiatry, 19, 528-537.

Ismail, B., Cantor-Graae, E. \& McNeil, T. F. (1998) Neurological abnormalities in schizophrenic patients and their siblings. American Journal of Psychiatry, $\mathbf{1 5 5}$ 84-89. 
Johnstone, E. C., Macmillan, J. F., Frith, C. D., et al (1990) Further investigation of the predictors of outcome following first schizophrenic episodes. British Journal of Psychiatry, 157, 182-189.

Kennard, M. A. (1960) Value of equivocal signs in neurologic diagnosis. Neurology, 10, 753-764.

King, D. J., Wilson, A., Cooper, S. J., et al (1991) The clinical correlates of neurological soft signs in chronic schizophrenia. British Journal of Psychiatry, 158, 770-775.

Kinney, D. K., Woods, B.T. \& Yurgelun-Todd, D. (1986) Neurological abnormalities in schizophrenic patients and their families. II. Neurologic and psychiatric findings in relatives. Archives of General Psychiatry, 43, 665-668.

Kolakowska, T., Williams, A. O., Jambor, K., et al (1985) Schizophrenia with good and poor outcome. III: Neurological 'soft' signs, cognitive impairment and their clinical significance. British Journal of Psychiatry, 146, 348-357.

Lane, A., Colgan, K., Moynihan, F., et al (1996) Schizophrenia and neurological soft signs: gender differences in clinical correlates and antecedent factors. Psychiatry Research, 64, 105-114.

Lawrie, S. M., Byrne, M., Miller, P., et al (200I) Neurodevelopmental indices and the development of psychotic symptoms in subjects at high risk of schizophrenia. British Journal of Psychiatry, 178 524-530.

Madsen, A. L., Vorstrup, S., Rubin, P., et al (1999) Neurological abnormalities in schizophrenic patients: a prospective follow-up study 5 years after first admission. Acta Psychiatrica Scandinavica, 100. $119-125$

Malesu, R. R., Cannon, M., Jones, P. B., et al (1996) Mixed-handedness in patients with functional psychosis. British Journal of Psychiatry, 168, 234-236.

Malla, A. K., Norman, R. M., Aguilar, O., et al (1997) Relationship between neurological 'soft signs' and syndromes of schizophrenia. Acta Psychiatrica Scandinavica, 96, 274-280.

Manschreck, T. C. \& Ames, D. (1984) Neurologic features and psychopathology in schizophrenic disorders. Biological Psychiatry, 19, 703-719.

_, Maher, B. A. \& Ader, D. N. (198I) Formal thought disorder, the type-token ratio and disturbed voluntary motor movement in schizophrenia. British Journal of Psychiatry, 139, 7-15.

, Rucklos, M. E., et al (1982) Disturbed voluntary motor activity in schizophrenic disorder. Psychological Medicine, 12, 73-84.

Marcus, J., Hans, S. L., Lewow, E., et al (1985) Neurological findings in high-risk children: childhood assessment and 5-year followup. Schizophrenia Bulletin, II, $85-100$
_ , _ , Auerbach, J. G., et al (1993) Children at risk for schizophrenia: The Jerusalem Infant Development Study: II. Neurobehavioral deficits at school age. Archives of General Psychiatry, 50, 797-809.

Mohr, F., Hubmann, W., Cohen, R., et al (1996) Neurological soft signs in schizophrenia: assessment and correlates. European Archives of Psychiatry and Clinical Neuroscience, 246, 240-248.

Mosher, L. R., Pollin, W. \& Stabenau, J. R. (197I) Identical twins discordant for schizophrenia. Neurologic findings. Archives of General Psychiatry, 24, 422-430.

Murray, R. M. (1994) Neurodevelopmental schizophrenia: The rediscovery of dementia praecox. British Journal of Psychiatry, 165 (suppl. 25), 6-12.

Niethammer, R., Weisbrod, M., Schiesser, S., et al (2000) Genetic influence on laterality in schizophrenia? A twin study of neurological soft signs. American Journal of Psychiatry, 157, 272-274.

Oldfield, R. C. (197I) The assessment and analysis of handedness: the Edinburgh inventory. Neuropsychologia, 9, 97-113

Orr, K. G., Cannon, M., Gilvarry, C. M., et al (1999) Schizophrenic patients and their first-degree relatives show an excess of mixed-handedness. Schizophrenia Research, 39, 167-176.

Quitkin, F., Rifkin, A. \& Klein, D. F. (1976) Neurologic soft signs in schizophrenia and character disorders. Organicity in schizophrenia with premorbid asociality and emotionally unstable character disorders. Archives of General Psychiatry, 33, 845-853.

Rieder, R. O. \& Nichols, P. L. (1979) Offspring of schizophrenics. III. Hyperactivity and neurological soft signs. Archives of General Psychiatry, 36 665-674.

Rochford, J. M., Detre, T., Tucker, G. J., et al (1970) Neuropsychological impairments in functional psychiatric diseases. Archives of General Psychiatry, 22. |14-119.

Rossi, A., De Cataldo, S., Di Michele V., et al (1990) Neurological soft signs in schizophrenia. British Journal of Psychiatry, 157, 735-739.

Rubin, P., Vostrup, S., Hemmingsen, R., et al (1994) Neurological abnormalities in patients with schizophrenia or schizophreniform disorder at first admission to hospital: correlations with computerized tomography and regional cerebral blood flow findings. Acta Psychiatrica Scandinavica, 90, 385-390.

Sanders, R. D., Keshavan, M. S. \& Schooler, N. R. (1994) Neurological examination abnormalities in neuroleptic-naive patients with first-break schizophrenia: preliminary results. American fournal of Psychiatry, 15I, 1231-1233.

Schroder, J., Niethammer, R., Geider, F. J., et al (1991) Neurological soft signs in schizophrenia. Schizophrenia Research, 6, 25-30.
Sharma, T., Lancaster, E., Sigmundsson, T., et al (1999) Lack of normal pattern of cerebral asymmetry in familial schizophrenic patients and their relatives The Maudsley Family Study. Schizophrenia Research, 40 III-120.

Stevens, J. R. (1982) Neurology and neuropathology of schizophrenia. In Schizophrenia as a Brain Disease (eds F. A. Henn \& H. A. Nasrallah), pp. II2-147. New York: Oxford University Press.

The Scottish Schizophrenia Research Group (1987) The Scottish First Episode Schizophrenia Study. I. Patient identification and categorisation. British Journal of Psychiatry, I50, 33I-333.

Torrey, E. G. (1980) Neurological abnormalities in schizophrenic patients. Biological Psychiatry, I5, 38I-388.

Tucker, G. J. \& Silberfarb, P. M. (1978) Neurologic dysfunction in schizophrenia: significance for diagnostic practice. In Psychiatric Diagnosis: Exploration of Biological Predictors (eds H. Akiskal \& W. Webb), pp. 453-462. New York: Spektrum.

_ , Campion, E.W., Kelleher, P. A., et al (1974) The relationship of subtle neurologic impairments to disturbances of thinking. Psychotherapy and Psychosomatics, 24, 165-169.

\& Silberfarb, P. M. (1975) Sensorimotor functions and cognitive disturbance in psychiatric patients. American Journal of Psychiatry, 132, 17-21.

Walker, E. \& Shaye, J. (1982) Familial schizophrenia. A predictor of neuromotor and attentional abnormalities in schizophrenia. Archives of General Psychiatry, 39. II53-II56.

Wegner, J.T., Catalano, F., Gibralter, J., et a (1985) Schizophrenics with tardive dyskinesia. Neuropsychological deficit and family psychopathology. Archives of General Psychiatry, 42, 860-865.

Weinberger, D. R. \& Wyatt, R. J. (1982) Cerebral ventricular biological marker for sub-typing chronic schizophrenia. In Biological Markers in Psychiatry and Neurology (ed. E. E. Usdin), pp. 505-512. Oxford: Pergamon Press.

Wong, A. H., Voruganti, L., Heslegrave, R. J., et al (1997) Neurologic abnormalities in schizophrenic patients and their families. I. Comparison of schizophrenic, bipolar, and substance abuse patients and normal controls. Archives of General Psychiatry, $\mathbf{4 3}$ 657-663.

Woods, B.T., Kinney, D. K. \& Yurgelun-Todd, D. (1986) Neurophrenic patients and their families. I. Comparison of schizophrenic, bipolar, and substance abuse patients and normal controls. Archives of General Psychiatry, 43, 657-663. 